\section{Human cytomegalovirus latency and reactivation in and beyond the myeloid lineage}

\author{
John Sinclair ${ }^{1} \&$ Emma Poole ${ }^{*, 1}$
}

\begin{abstract}
After primary infection with human cytomegalovirus (HCMV), which rarely causes any serious clinical problems in the immune competent, the virus persists subclinically for the lifetime of the host due, at least in part, to its ability to undergo latent infection. By contrast, HCMV can be a serious cause of morbidity, and in some cases mortality, upon primary infection of, or reactivation in, immune suppressed individuals. While current antivirals that target its lytic lifecycle have helped enormously in managing HCMV disease, to date, there are no available antivirals that target latent infection. In this review, we discuss research using natural and experimental models of latency that has led to some understanding of how HCMV latency is maintained, and reactivation controlled, in the myeloid lineage. Such analyses are now beginning to inform us of novel rationales that could allow the development of novel antivirals to target latency, itself.
\end{abstract}

Human cytomegalovirus (HCMV) is the prototypic $\beta$-herpesvirus and, like all herpesviruses, establishes a life-long persistent infection in the host [1]. This carriage of virus for the lifetime of the infected individual likely reflects a complex interplay between two forms of virus lifecycle in vivo: low level lytic replication (which is too low to permit routine detection of infectious virons) as well as latent carriage - biological property of all herpesviruses. In contrast with lytic infection, during latent infection, there is a restricted viral gene expression profile that results in a lack of lytic replication and an absence of any virion production.

In healthy immune competent virus carriers, it is argued that any low level lytic replication is robustly controlled by the immune system and is, hence, routinely asymptomatic [1]. However, in immune-compromised individuals, such low level virus production is no longer adequately controlled and leads to detectable lytic infection of numerous cell types resulting in overt disease in an array of tissues [2-5].

There is currently no robust vaccine for HCMV and, although current antiviral treatments have been very effective in a number of clinical settings, all licensed antivirals for HCMV, to date, target lytic replication and will not target latently infected cells in which there is a lack of virus replication and production. Consequently, identifying and understanding the cell types that HCMV is carried in during latency and understanding lytic-latent regulation is crucial to allow the development of novel antiviral therapeutics to comprehensively target this persistent human pathogen.

\section{HCMV latent carriage in cells of the myeloid lineage}

Hints that helped identify the sites of HCMV latency in asymptomatic seropositive carriers have come from the observations that infectious virus can be transmitted from healthy HCMV seropositive blood donors to seronegative immune suppressed recipients (as well as seropositive recipients

'Department of Medicine, Box 157, University of Cambridge, Addenbrooke's Hospital, Hills Road, Cambridge, CB2 0QQ, UK *Author for correspondence: elp27@hermes.cam.ac.uk

\section{KEYWORDS}

- human cytomegalovirus

- latency • myeloid

- reactivation 
infected with a different strain of HCMV, e.g., from a seropositive allograft) that can, after immune suppression, result in overt HCMV disease in these recipient patients [6-8]. However, infectious virus cannot be isolated from the blood of healthy donors [9], suggesting a latent carriage of the virus in the blood compartment. The exact cells involved were further defined by the observation that transfusion of leukocytedepleted blood substantially decreased HCMV disease [10], arguing that infectious virus was transmitted in the white blood cell compartment. The advent of sensitive DNA PCR techniques confirmed that HCMV DNA is present in peripheral blood mononuclear cells PBMC [11-14] but specifically in monocytes, not $\mathrm{T}$ or $\mathrm{B}$ cells [14], and also present in their $\mathrm{CD} 4^{+}$progenitors isolated from bone marrow [15]. Studies of naturally latently infected peripheral blood monocytes and $\mathrm{CD}_{3} 4^{+}$progenitors derived from bone marrow or from G-CSF mobilized peripheral blood also showed that the presence of viral genome in monocytes and $\mathrm{CD} 34^{+}$progenitor cells was in the absence of extensive lytic gene transcription, suggesting that that these cells may represent true sites of latent infection [14-16]. In these early studies, viral DNA copy number was shown to be extremely low with frequencies around one latently infected cell in $10^{4} / 10^{5}$ [17]. However, there remains a need for follow-on studies to analyze in detail the full spectrum of myeloid cells that harbor latent viral genome.

\section{$\mathrm{HCMV}$ reactivation by differentiation of myeloid progenitor cells}

Clearly, undifferentiated myeloid cells carry the latent HCMV genome in vivo. However, a crucial criterion for a biologically relevant site of latency is the ability of latent virus to reactivate. This leads to the important question of the signals involved in reactivating HCMV from these myeloid sites of latency.

Insights into these signals came from the observations that, while experimental infection of monocytes was routinely nonpermissive, differentiation of monocytes to monocyte-derived macrophages led to a fully permissive phenotype $[18,19]$. This suggested that the differentiation status of the myeloid lineage might be critically important for determining whether or not infection becomes lytic or latent. Consistent with this, subsequent analyses have shown that ex vivo differentiation of naturally latently infected peripheral blood monocytes or CD34 ${ }^{+}$ progenitors to monocyte-derived macrophages or dendritic cells resulted in reactivation of lytic gene expression from latent genomes [14] and the production of infectious virions [20,21]. Also, the observation that DCs isolated directly from peripheral blood show evidence of endogenous immediate early RNA expression fits well with the argument that myeloid cells are, indeed, sites of HCMV reactivation in vivo [22]. However, the exact myeloid cell types that efficiently carry and reactivate HCMV in vivo will require a much more detailed analysis of naturally latent myeloid cells, perhaps using combinations of specific myeloid cell markers. Unfortunately, as discussed above, the frequency of naturally latent cells in the peripheral blood makes these types of analyses beyond our current technology and so many laboratories have resorted to the use of infection of primary cells [21,23-28], as well as established cells lines [29-31] in culture as models of experimentally latent infection. In all cases, these models allow the long-term culture (up to 20 days for primary myeloid cells) of cells carrying quiescent viral genomes from which virus can be reactivated after differentiation along the myeloid lineage. In many cases, these experimental models of latency faithfully reflect a number of aspects of natural latency [21] but, importantly, much of the data accrued from such experimental infection models still needs to be cross-confirmed in natural infection.

\section{Chromatin modulates HCMV latency $\&$ reactivation in the myeloid cells}

The experimental models outlined above have been used to analyze the mechanisms that control latency and reactivation in the myeloid lineage. It is now accepted that these mechanisms, which result in HCMV remaining latent in myeloid progenitors but that result in virus reactivation following myeloid differentiation, appear to be closely linked to the chromatin structure of the major immediate early promoter (MIEP), which drives immediate early lytic gene expression [21,32]. Essentially, during latency the MIEP is associated with histone repressive marks likely resulting from binding of high levels of repressive transcription factors that are differentiation dependent; although, so far, these have only been identified by transfection analyses of the viral MIEP in undifferentiated and differentiated cells types [22,33-37]. It is likely that as progenitor cells differentiate, negative regulators of the MIEP decrease 
and positive regulators increase, resulting in the MIEP becoming associated with markers of active chromatin [21,32], consistent with the observed expression of lytic genes in these cells. Importantly, this link between histone post-translational modification and the regulation of HCMV latency and reactivation is also observed in naturally latent cells ex vivo [22]. Whether the virus is involved in the differentiation events required for its reactivation, or just a silent passenger waiting for extrinsic signals to occur before reactivation can occur, is not entirely clear. Clearly, the experimental addition of cytokines to naturally latent myeloid progenitor cells results in differentiation and concomitant virus reactivation. However, transcriptome analyses of infected monocytes also suggest that HCMV infection, in itself, can modulate the differentiation status of these cells [38]. Therefore, it is likely that both virus-driven effects, as well as extrinsic cellular factors, play a role in the regulation of HCMV latency and reactivation.

Regardless, analyses of experimentally latent myeloid cells, as well as naturally latent cells, ex vivo, have consistently implicated chromatinmediated modulation of the viral MIEP in the control of viral lytic gene expression during latency and reactivation and the view that the differentiation-specific milieu of cellular, and perhaps viral, transcription factors, plays an important role in this modulation. For instance, recent reports using experimental models of latency have suggested that the suppression of viral lytic gene expression during latent infection may be facilitated by the absence of the viral transcriptional activator pp71 [39], as well as latency-associated expression of a viral long ncRNA (lnc4.9 RNA) [40]. Thus, it appears that the reactivation from latency is probably regulated by both external factors contributing to the differentiation status of the myeloid cell, as well as viral gene products present during latent infection.

\section{HCMV genome maintenance in the myeloid lineage}

CD $34^{+}$hematopoietic stem cells, then clearly carry latent and, importantly, reactivatable viral genomes and it is likely that this is carried as an episome [41]. However, how this is maintained for the lifetime of the infected host in unclear. It is possible that an initial primary infection is set up in an extremely long-lived pluripotent hematopoeitic progenitor. While such very long-lived progenitor populations are believed to exist in vivo [42], it seems unlikely that a single initial infection provides sufficient latently infected hematopoietic progenitors for the lifetime of the host. We favor the view that, in normal healthy HCMV seropositive carriers, subclinical reactivation routinely occurs during persistent carriage and these, or multiple subclinical reinfections, continually reseed new hematopoietic progenitors during the life of the host. Nevertheless, we cannot formally rule out that a self-renewing hematopoetic progenitor population maintains a latently replicating form of the viral genome. Although there is no direct evidence for such a mechanism of latent viral genome maintenance, a recent analysis of transcripts during natural HCMV latency detected transcripts associated with viral DNA replication and proteins encoded by these RNAs could associate with viral genomes from experimentally latently infected cells [42]. Attractively, in these studies, the terminal repeat region of the viral genome appeared to provide a cis-acting genome maintenance function [40]. However, many of the other viral transcripts identified also encode viral gene products that are generally thought to be involved in lytic viral DNA replication. Consequently, it is unclear at present how these observations might fit with a latent genome replication model.

Another important question about latent viral genome carriage is posed by the observation that pleuripotent CD $34^{+}$cells carry latent viral genome, yet latent viral genomes are only carried down the myeloid lineage - it is not present in lymphoid derivatives [14]. A hint as to how this might be explained comes from studies that are consistent with the view that latent infection may result in changes in cellular gene expression that favor myeloid differentiation [43-47]. For instance, we have shown recently that a number of changes in cellular gene expression result from latent infection. One of these is an increase in the cellular transcription factor GATA-2, which is known to be involved in maintaining myeloid differentiation $[43,44]$. This, again, suggests that latent infection, itself, may help drive latently infected undifferentiated cells down the myeloid lineage resulting in HCMV reactivating in important antigen-presenting cells (such as dendritic cells) where it can modulate multiple arms of the host immune response. 
Viral \& cellular gene regulation by HCMV in the myeloid lineage

To what extent HCMV is a silent passenger in latently infected myeloid cells has long been debated. Recent evidence now strongly suggests that latent infection, during which there is a much more limited viral transcription profile, is still able to extensively manipulate the cell. Although there are discrepancies in the literature regarding the specific viral genes expressed during latency, likely due to differences in the model systems used and the sensitivity of detection techniques, expression of a number of viral genes is routinely detectable in naturally latent infected cells. These include, US28, latent viral IL-10, UL144, LUNA and UL138 [45,48-50]. Although the exact roles of the products of these genes during HCMV latency have not been fully established, some insights into their possible roles during latent infection have been determined. For instance, UL138 has been suggested to be important for maintenance of latent infection either as a factor required for latent carriage or as a factor to suppress reactivation - at least in some experimental latent models [49]; the latent viral IL-10 can cause downregulation of MHC class II on the cell surface of cells [51]; LUNA has been reported to be important for reactivation from latency [52]; US28 is a chemokine receptor that responds to CC chemokines and is also constitutively active [53] and UL144 is a TNF family ligand for HVEM $[54,55]$ that shows an ability to indirectly enhance the TH2 immune response in model systems and, thus, may act to suppress the TH1 immune response and avoid immune detection [56].

Interestingly, at least two of these latencyassociated genes (LUNA and UL144) have GATA-2 binding sites in their promoters [45,57] and suggest that the latency-associated increase in GATA-2, described above, may be dually functional; it not only helps drive the latently infected cell down the myeloid lineage but it is concomitantly used to drive viral latent gene expression [45]. While this suggests that any myeloid-active promoter may drive expression of any viral gene in the HCMV genome during latent infection, it has been shown that the insertion of a heterologous myeloid-active promoter in the HCMV genome is not sufficient, in itself, to lead to its transcriptional activation in myeloid cells [58]. Consequently, the exact mechanisms regulating viral latency-associated gene expression in early myeloid progenitors is likely more complex than simply the presence or absence of a myeloid-active promoter.

Latent infection of myeloid cells also has a profound effect on cellular gene expression. For example, latent infection of $\mathrm{CD} 34^{+}$cells is known to manipulate the expression of a number of cellular miRNAs and at least one of these changes was involved in the induction of expression of GATA-2 [46]. Similarly, a recent analysis of secreted cellular proteins during latent infection showed major changes in the latency-associated secretome and that this directly affected $\mathrm{CD}^{+} \mathrm{T}$ cell surveillance of latently infected cells [59], as well as the maintenance of latent viral genome by inhibiting apoptosis [46].

Therefore, although far from complete, a picture is slowly emerging of how HCMV viral gene expression is regulated during latency; how latency-associated viral gene expression modulates cellular gene expression and how this orchestration of viral and cellular gene expression optimizes latent carriage and reactivation in the myeloid lineage.

The importance of understanding such virushost interactions during latency cannot be overestimated and this has recently been underscored by the fact that changes in cellular gene expression resulting from latency-associated expression of the viral UL138 gene product has led to the first example of a novel therapeutic strategy to target latently infected cells [60].

\section{HCMV carriage in other cell types?}

GATA2 is not only important for myeloid differentiation but also differentiation of hematopoietic progenitors along the endothelial cell lineage $[44,61]$. Some endothelial cells can be derived from the same cell progenitors as myeloid cells $[62,63]$. Further to this, HCMV-associated pathologies can occur in endothelial cells of the lung and intestine [64]. These observations have led to the suggestion that HCMV may also be carried along the endothelial cell lineage [65]. However, an initial study analyzing endothelial cells from the saphenous vein of healthy seropositive individuals did not detect HCMV [66], which suggested that endothelial cells of the microvasculature are unlikely to be a site of HCMV latency. It is, however, possible that aortic endothelial cells of the macrovasculature may harbor latent HCMV. This does warrant further investigation but the ethical issues of analyzing such tissues from healthy individuals makes addressing this problematic. 
Recent studies that have analyzed differential permissiveness of differentiating neural cell populations have also implicated neural progenitor cells as potential reservoirs of virus and viral persistence $[67,68]$ but whether these are true sites of latency in vivo will require detailed further analysis of naturally latent cells and, importantly, that these cells can be induced to reactivate latent quiescent genomes.

\section{Conclusion \& future perspective}

Our understanding of HCMV latency has slowly grown over the last decade and detailed analyses of the effects of latent infection on the cell has, at least in one case, led to the possibility of novel therapeutics to target myeloid cells carrying latent viral genome [60] and, as our understanding of changes in the latent cell phenotype increases, this is likely to expand significantly over the next 5-10 years.
However, if HCMV is ever to be fully controlled in the clinical setting, an even more complete understanding of where latent genomes reside, perhaps in sites other than the myeloid lineage, how these genomes are maintained and the roles of latency-associated gene expression, as well as the effects of latent infection on the cell, will be essential.

\section{EXECUTIVE SUMMARY}

\section{Human cytomegalovirus latent carriage in cells of the myeloid lineage}

- The myeloid lineage is a site of latency.

\section{Human cytomegalovirus reactivation by differentiation of myeloid progenitor cells}

- There are a number of factors that regulate reactivation from latency.

\section{Chromatin modulates human cytomegalovirus latency \& reactivation in the myeloid cells}

- Chromatin structure plays a role in the regulation of latency and reactivation.

\section{Human cytomegalovirus genome maintenance in the myeloid lineage}

- Viral genome is carried in cells of the myeloid lineage.

\section{Viral \& cellular gene regulation by human cytomegalovirus in the myeloid lineage}

- Only a few genes have been demonstrated to be transcribed during human cytomegalovirus latency.

- Roles for viral and cellular genes during latency in myeloid cells.

Human cytomegalovirus carriage in other cell types?

- There is evidence to suggest that human cytomegalovirus is not only carried in the myeloid lineage.

\section{References}

1 Rook AH. Interactions of cytomegalovirus with the human immune system. Rev. Infect. Dis. 10(Suppl. 3), S460-S467 (1988).

2 Zaia JA. The biology of human cytomegalovirus infection after bone marrow transplantation. Int. J. Cell Cloning 4(Suppl. 1), 135-154 (1986).

3 Orloff SL, Hwee YK, Kreklywich C et al. Cytomegalovirus latency promotes cardiac lymphoid neogenesis and accelerated allograft rejection in CMV naive recipients. Am. J. Transplant. 11(1), 45-55 (2012).
4 Comar M, Delbue S, Lepore L et al. Latent viral infections in young patients with inflammatory diseases treated with biological agents: prevalence of JC virus genotype 2. J. Med. Virol. 85(4), 716-722 (2013).

5 Sissons JG, Sinclair JH, Borysiewicz LK. Pathogenesis of human cytomegalovirus disease and the kidney. Kidney Int. Suppl. 35, S8-S12 (1991).

6 Yeager AS. Transfusion-acquired cytomegalovirus infection in newborn infants. Am. J. Dis. Child. 128(4), 478-483 (1974).
7 Adler SP. Transfusion-associated cytomegalovirus infections. Rev. Infect. Dis. 5(6), 977-993 (1983).

8 Tolpin MD, Stewart JA, Warren D et al. Transfusion transmission of cytomegalovirus confirmed by restriction endonuclease analysis. J. Pediatr. 107(6), 953-956 (1985).

9 Jordan MC. Latent infection and the elusive cytomegalovirus. Rev. Infect. Dis. 5(2), 205-215 (1983).

10 Hansen LL, Nieuwenhuis I, Hoffken G, Heise W. [Retinitis in AIDS patients: diagnosis, follow-up and treatment]. Fortschr. Ophthalmol. 86(3), 232-238 (1989). 
11 Schrier RD, Nelson JA, Oldstone MB. Detection of human cytomegalovirus in peripheral blood lymphocytes in a natural infection. Science 230(4729), 1048-1051 (1985).

12 Stanier P, Taylor DL, Kitchen AD, Wales N, Tryhorn Y, Tyms AS. Persistence of cytomegalovirus in mononuclear cells in peripheral blood from blood donors. BMJ 299(6704), 897-898 (1989).

13 Bevan IS, Daw RA, Day PJ, Ala FA, Walker MR. Polymerase chain reaction for detection of human cytomegalovirus infection in a blood donor population. Br. J. Haematol. 78(1), 94-99 (1991).

14 Taylor-Wiedeman J, Sissons JG, Borysiewicz LK, Sinclair JH. Monocytes are a major site of persistence of human cytomegalovirus in peripheral blood mononuclear cells. J. Gen. Virol. 72 (Pt 9), 2059-2064 (1991).

15 Mendelson M, Monard S, Sissons P, Sinclair J. Detection of endogenous human cytomegalovirus in CD34+ bone marrow progenitors. J. Gen. Virol. 77 (Pt 12), 3099-3102 (1996).

16 Larsson S, Soderberg-Naucler C, Wang FZ, Moller E. Cytomegalovirus DNA can be detected in peripheral blood mononuclear cells from all seropositive and most seronegative healthy blood donors over time. Transfusion 38(3), 271-278 (1998).

17 Slobedman B, Mocarski ES. Quantitative analysis of latent human cytomegalovirus. J. Virol. 73(6), 4806-4812 (1999).

18 Ibanez CE, Schrier R, Ghazal P, Wiley C, Nelson JA. Human cytomegalovirus productively infects primary differentiated macrophages. J. Virol. 65(12), 6581-6588 (1991).

19 Lathey JL, Spector SA. Unrestricted replication of human cytomegalovirus in hydrocortisonetreated macrophages. J. Virol. 65, 6371-6375 (1991).

20 Soderberg-Naucler C, Fish KN, Nelson JA. Reactivation of latent human cytomegalovirus by allogeneic stimulation of blood cells from healthy donors. Cell 91(1), 119-126 (1997).

21 Reeves MB, Lehner PJ, Sissons JG, Sinclair $\mathrm{JH}$. An in vitro model for the regulation of human cytomegalovirus latency and reactivation in dendritic cells by chromatin remodelling. J. Gen. Virol. 86(Pt 11), 2949-2954 (2005).

22 Reeves MB, Macary PA, Lehner PJ, Sissons JG, Sinclair JH. Latency, chromatin remodeling, and reactivation of human cytomegalovirus in the dendritic cells of healthy carriers. Proc. Natl Acad. Sci. USA 102(11), 4140-4145 (2005).
23 Maciejewski JP, Bruening EE, Donahue RE, Mocarski ES, Young NS, St Jeor SC. Infection of hematopoietic progenitor cells by human cytomegalovirus. Blood 80 (1), 170-178 (1992).

24 Kondo K, Kaneshima H, Mocarski ES. Human cytomegalovirus latent infection of granulocyte-macrophage progenitors. Proc. Natl Acad. Sci. USA 91(25), 11879-11883 (1994).

25 Minton EJ, Tysoe C, Sinclair JH, Sissons JG. Human cytomegalovirus infection of the monocyte/macrophage lineage in bone marrow. J. Virol. 68(6), 4017-4021 (1994).

26 Hahn G, Jores R, Mocarski ES. Cytomegalovirus remains latent in a common precursor of dendritic and myeloid cells. Proc. Natl Acad. Sci. USA 95(7), 3937-3942 (1998).

27 Goodrum FD, Jordan CT, High K, Shenk T. Human cytomegalovirus gene expression during infection of primary hematopoietic progenitor cells: a model for latency. Proc. Natl Acad. Sci. USA 99(25), 16255-16260 (2002).

28 Hargett D, Shenk TE. Experimental human cytomegalovirus latency in CD14 ${ }^{+}$ monocytes. Proc. Natl Acad. Sci. USA 107(46), 20039-20044 (2011).

29 Albright ER, Kalejta RF. Myeloblastic cell lines mimic some but not all aspects of human cytomegalovirus experimental latency defined in primary $\mathrm{CD} 34^{+}$cell populations. J. Virol. 87(17), 9802-9812 (2013).

30 Keyes LR, Bego MG, Soland M, St Jeor S. Cyclophilin A is required for efficient human cytomegalovirus DNA replication and reactivation. J. Gen. Virol. 93(Pt 4), 722-732 (2012).

31 Penkert RR, Kalejta RF. Human embryonic stem cell lines model experimental human cytomegalovirus latency. MBio 4(3), e00298-e00213 (2013).

32 Murphy EA, Streblow DN, Nelson JA, Stinski MF. The human cytomegalovirus IE86 protein can block cell cycle progression after inducing transition into the $S$ phase of permissive cells. J. Virol. 74(15), 7108-7118 (2000).

33 Murphy Jc FW, Verdin E, Sinclair JH. Control of cytomegalovirus lytic gene expression by histone acetylation. $E M B O$ J. 21, 1112-1120 (2002).

34 Thomas MJ, Seto E. Unlocking the mechanisms of transcription factor YY1: are chromatin modifying enzymes the key? Gene 236(2), 197-208 (1999).
35 Pizzorno MC. Nuclear cathepsin B-like protease cleaves transcription factor YY1 in differentiated cells. Biochim. Biophys. Acta 1536(1), 31-42 (2001).

36 Bain M, Mendelson M, Sinclair J. Ets-2 Repressor Factor (ERF) mediates repression of the human cytomegalovirus major immediate-early promoter in undifferentiated non-permissive cells. J. Gen. Virol. 84(Pt 1), 41-49 (2003).

37 Wright E, Bain M, Teague L, Murphy J, Sinclair J. Ets-2 repressor factor recruits histone deacetylase to silence human cytomegalovirus immediate-early gene expression in non-permissive cells. J. Gen. Virol. 86(Pt 3), 535-544 (2005).

38 Chan G, Bivins-Smith ER, Smith MS, Smith PM, Yurochko AD. Transcriptome analysis reveals human cytomegalovirus reprograms monocyte differentiation toward an M1 macrophage. J. Immunol. 181(1), 698-711 (2008).

39 Saffert RT, Penkert RR, Kalejta RF. Cellular and viral control over the initial events of human cytomegalovirus experimental latency in CD34 ${ }^{+}$cells. J. Virol. 84(11), 5594-5604 (2013).

40 Rossetto CC, Tarrant-Elorza M, Pari GS. Cis and trans acting factors involved in human cytomegalovirus experimental and natural latent infection of CD14 $\left(^{+}\right)$monocytes and CD34 (') cells. PLoS Pathog. 9(5), e1003366 (2013).

41 Bolovan-Fritts CA, Mocarski ES, Wiedeman JA. Peripheral blood CD14(+) cells from healthy subjects carry a circular conformation of latent cytomegalovirus genome. Blood 93(1), 394-398 (1999).

42 Miyamoto T. Role of osteoclasts in regulating hematopoietic stem and progenitor cells. World J. Orthop. 4(4), 198-206 (2013).

43 Tsai FY, Orkin SH. Transcription factor GATA-2 is required for proliferation/survival of early hematopoietic cells and mast cell formation, but not for erythroid and myeloid terminal differentiation. Blood 89(10), 3636-3643 (1997).

44 De Pater E, Kaimakis P, Vink CS et al. Gata2 is required for HSC generation and survival. J. Exp. Med. 210 (13), 2843-2850 (2013).

45 Poole E, Walther A, Raven K, Benedict CA, Mason GM, Sinclair J. The myeloid transcription factor GATA-2 regulates the viral UL144 gene during human cytomegalovirus latency in an isolate-specific manner. J. Virol. 87(8), 4261-4271 (2013).

46 Poole E, Mcgregor Dallas SR, Colston J, Joseph RS, Sinclair J. Virally induced changes 
in cellular microRNAs maintain latency of human cytomegalovirus in CD34 progenitors. J. Gen. Virol. 92(Pt 7), 1539-1549 (2011).

47 Reeves MB, Breidenstein A, Compton T. Human cytomegalovirus activation of ERK and myeloid cell leukemia-1 protein correlates with survival of latently infected cells. Proc. Natl Acad. Sci. USA 109(2), 588-593 (2012).

48 Bego M, Maciejewski J, Khaiboullina S, Pari G, St Jeor S. Characterization of an antisense transcript spanning the UL81-82 locus of human cytomegalovirus. J. Virol. 79(17), 11022-11034 (2005).

49 Goodrum F, Reeves M, Sinclair J, High K, Shenk T. Human cytomegalovirus sequences expressed in latently infected individuals promote a latent infection in vitro. Blood 110(3), 937-945 (2007).

50 Jenkins C, Abendroth A, Slobedman B. A novel viral transcript with homology to human interleukin-10 is expressed during latent human cytomegalovirus infection. J. Virol. 78(3), 1440-1447 (2004).

51 Cheung AK, Gottlieb DJ, Plachter B et al. The role of the human cytomegalovirus UL111A gene in down-regulating $\mathrm{CD}^{+}$ T-cell recognition of latently infected cells: implications for virus elimination during latency. Blood 114(19), 4128-4137 (2009).

52 Keyes LR, Hargett D, Soland $\mathrm{M}$ et al. HCMV protein LUNA is required for viral reactivation from latently infected primary CD14(+) cells. PLoS ONE 7(12), e52827 (2013).

53 Neote K, Digregorio D, Mak JY, Horuk R, Schall TJ. Molecular cloning, functional expression, and signaling characteristics of a C-C chemokine receptor. Cell 72(3), 415-425 (1993).
54 Benedict CA, Butrovich KD, Lurain NS et al. A novel viral TNF receptor superfamily member in virulent strains of human cytomegalovirus. J. Immunol. 162, 69676970 (1999).

55 Cheung TC, Humphreys IR, Potter KG et al. Evolutionary divergent herpesviruses modulate $T$ cell activation by targeting the herpesvirus entry mediator cosignaling pathway. Proc. Natl Acad. Sci. USA 37, 13218-13223. (2005).

56 Poole E, King CA, Sinclair JH, Alcami A. The UL144 gene product of human cytomegalovirus activates NFkappaB via a TRAF6-dependent mechanism. EMBO J. 25(18), 4390-4399 (2006).

57 Reeves MB, Sinclair JH. Analysis of latent viral gene expression in natural and experimental latency models of human cytomegalovirus and its correlation with histone modifications at a latent promoter. J. Gen. Virol. 91(Pt 3), 599-604 (2010).

58 Qin Q, Penkert RR, Kalejta RF. Heterologous viral promoters incorporated into the human cytomegalovirus genome are silenced during experimental latency. J. Virol. 87(17), 9886-9894 (2013).

59 Mason GM, Poole E, Sissons JG, Wills MR, Sinclair JH. Human cytomegalovirus latency alters the cellular secretome, inducing cluster of differentiation (CD) $4^{+}$T-cell migration and suppression of effector function. Proc. Natl Acad. Sci. USA 109(36), 14538-14543 (2012).

60 Weekes MP, Tan SY, Poole E et al. Latencyassociated degradation of the MRP1 drug transporter during latent human cytomegalovirus infection. Science 340(6129), 199-202 (2013).

61 Lui KO, Zangi L, Silva EA et al. Driving vascular endothelial cell fate of human multipotent Isl1 $1^{+}$heart progenitors with VEGF modified mRNA. Cell Res. 23(10), 1172-1186 (2013).

62 Goodell MA, Brose K, Paradis G, Conner AS, Mulligan RC. Isolation and functional properties of murine hematopoietic stem cells that are replicating in vivo. J. Exp. Med. 183(4), 1797-1806 (1996).

63 Quirici N, Soligo D, Caneva L, Servida F, Bossolasco P, Deliliers GL. Differentiation and expansion of endothelial cells from human bone marrow $\mathrm{CD} 133\left({ }^{+}\right)$cells. $\mathrm{Br}$. J. Haematol. 115(1), 186-194 (2001).

64 Sinzger C, Grefte A, Plachter B, Gouw AS, The TH, Jahn G. Fibroblasts, epithelial cells, endothelial cells and smooth muscle cells are major targets of human cytomegalovirus infection in lung and gastrointestinal tissues. J. Gen. Virol. 76 (Pt 4), 741-750 (1995).

65 Jarvis MA, Nelson JA. Human cytomegalovirus persistence and latency in endothelial cells and macrophages. Curr. Opin. Microbiol. 5(4), 403-407 (2002).

66 Reeves MB, Coleman H, Chadderton J, Goddard M, Sissons JG, Sinclair JH. Vascular endothelial and smooth muscle cells are unlikely to be major sites of latency of human cytomegalovirus in vivo. J. Gen. Virol. 85(Pt 11), 3337-3341 (2004).

67 Belzile JP, Stark TJ, Yeo GW, Spector DH. Human cytomegalovirus infection of human embryonic stem cell-derived primitive neural stem cells is restricted at several steps but leads to the persistence of viral DNA. J. Virol. 88(8),4021-4039 (2014).

68 Duan YL, Ye HQ, Zavala AG et al. Maintenance of large numbers of virus genomes in human cytomegalovirus-infected T98G glioblastoma cells. J. Virol. 88(7), 3861-3873 (2014). 